\title{
Éthique environnementale et développement durable : Réflexions sur le Principe Responsabilité de Hans Jonas
}

Environmental ethics and sustainable development: Reflections on Hans Jonas' Responsibility Principle

\section{Sylvie Ferrari}

\section{OpenEdition \\ Journals}

Édition électronique

URL : http://journals.openedition.org/developpementdurable/8441

DOI : 10.4000/developpementdurable.8441

ISSN : 1772-9971

Éditeur

Association DD\&T

Référence électronique

Sylvie Ferrari, «Éthique environnementale et développement durable : Réflexions sur le Principe

Responsabilité de Hans Jonas », Développement durable et territoires [En ligne], Vol. 1, n 3 | Décembre 2010, mis en ligne le 07 décembre 2010, consulté le 30 avril 2019. URL : http://

journals.openedition.org/developpementdurable/8441; DOI : 10.4000/developpementdurable.8441

Ce document a été généré automatiquement le 30 avril 2019

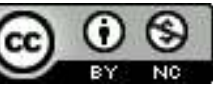

Développement Durable et Territoires est mis à disposition selon les termes de la licence Creative Commons Attribution - Pas d'Utilisation Commerciale 4.0 International. 


\title{
Éthique environnementale et développement durable : Réflexions sur le Principe Responsabilité de Hans Jonas
}

\author{
Environmental ethics and sustainable development: Reflections on Hans Jonas' \\ Responsibility Principle
}

Sylvie Ferrari

1 Le concept de développement durable tel que le définit le rapport Brundtland (WCED, 1987) considère la question de la justice intergénérationnelle comme une nécessité dans une perspective de long terme. En le définissant comme un développement «qui répond aux besoins du présent sans compromettre la capacité des générations futures de répondre aux leurs ", la préservation de l'environnement apparaît alors intrinsèquement liée à la dynamique du développement des sociétés. L'éthique environnementale entre dans le champ de l'économie et suggère que la répartition des ressources et leur accès sont indissociables des impacts environnementaux résultant de certains usages irréfléchis (surexploitation, dégradation) des ressources sur un temps long. Si la dimension intragénérationnelle n'est pas ici directement concernée, il n'en demeure pas moins que la possibilité d'accéder ou non à certaines ressources (quantité et qualité) conditionne la dynamique de développement et donc influence le niveau de bien-être des générations présentes. Sur ce point, il est de plus en plus évident que la répartition des richesses dans une société peut influencer fortement la protection des ressources environnementales (Mikkelson et al., 2007 ; Gagnon et al., 2008).

2 Dans une acception large, l'éthique environnementale peut être définie comme l'étude des liens entre l'espèce humaine et les autres espèces vivantes et inanimées de la biosphère. Ce champ de réflexion questionne les fondements moraux des rapports entre l'homme et la nature et analyse les implications sociétales en termes de devoirs et d'obligations selon deux angles d'approche : l'un de nature biocentrée et l'autre de nature 
anthropocentrée (Larrère, 1997 ; Larrère, Larrère, 1997). Cette dernière considère la protection des éléments de la nature (biodiversité, espaces naturels, ressources) en incluant également la question de leur transmission aux générations futures, tandis que l'approche biocentrée étend à tous les êtres vivants (animaux, plantes, écosystèmes...) la considération morale. Selon le positionnement retenu, les implications pour la protection des ressources peuvent être différentes.

Cet article poursuit deux objectifs. Le premier consiste à appréhender le développement durable sous l'angle de l'éthique environnementale et d'en dégager quelques enjeux majeurs pour la protection des ressources environnementales. Considérant la nécessité d'introduire le temps long, le second objectif vise à montrer comment le principe Responsabilité de Jonas peut modifier les décisions économiques en prenant en compte leurs effets potentiels sur les ressources environnementales et, indirectement, sur la durabilité du développement.

\section{Développement durable, équité entre générations et protection des ressources naturelles}

\subsection{Nature et durabilité}

4 Le contenu du concept de développement durable peut être approché à partir de trois dimensions interdépendantes - économique, sociale et environnementale - ayant chacune une finalité qui lui est propre. La dimension économique s'exprime par une combinaison optimale des ressources naturelles, humaines et techniques dans le but d'assurer la maximisation du bien-être des générations sur un horizon de long terme ; sa finalité réside dans l'absence de tout gaspillage de ressources (efficacité des allocations). La dimension sociale concerne l'accès aux ressources et leur répartition dans l'espace (niveau intragénérationnel) et dans le temps (niveau intergénérationnel); sa finalité est d'assurer l'équité entre les générations dans la distribution des dotations disponibles. Quant à la dimension environnementale, elle a trait à la gestion des stocks de ressources et à la préservation de leur qualité afin de d'assurer la permanence du capital naturel dans le temps.

5 La difficulté de la mise en œuvre du développement durable réside dans le niveau de compatibilité entre les trois finalités. Cela étant, si le concept de durabilité autorise des passerelles entre les phénomènes économiques et la nature, c'est par le biais de l'éthique : les générations sont liées entre elles par un socle commun, les ressources environnementales existant à un moment donné du temps, et la nature des liens dépend essentiellement de la manière dont les différents éléments naturels (marchands et non marchands) contribuent au bien-être des générations successives. Par conséquent, il ne peut y avoir de durabilité du développement des sociétés sans la prise en compte d'une éthique environnementale.

6 En général, le concept de durabilité appliqué au développement traduit la nécessité d'assurer la non-décroissance dans le temps du niveau de bien être pour les générations successives. Le bien-être, appréhendé par le prisme des besoins, doit être précisé ici. En effet, l'interprétation de la définition du développement durable à partir du rapport Brundtland conduit à retenir une approche minimaliste à la fois du concept de besoin en tant que besoin de base et de l'équité intergénérationnelle (Gosseries, 2008 ; Dasgupta, 
2008). L'exigence de durabilité ici repose sur une équité intergénérationnelle à contenu restreint, c'est-à-dire qui n'engage les générations présentes à l'égard des générations futures que pour garantir la couverture des besoins de base dans la perspective de maintenir dans le temps un niveau de bien-être non décroissant, et rien de plus.

Dans ce contexte, le problème économique à traiter d'un point de vue théorique est celui de la répartition intertemporelle des droits d'usage des ressources environnementales (à travers les stocks et services fournis par différentes fonctions assurées par la biosphère et les écosystèmes) sous la contrainte de satisfaire les besoins de base (ou besoins fondamentaux) des générations successives, alors que certaines ressources se raréfient et/ou se détériorent. Cette perspective dynamique conduit à mettre l'accent sur la dualité éminemment normative du développement durable qui combine deux éléments indissociables, l'optimalité parétienne et l'équité intergénérationnelle. Si la préservation de la qualité de certaines ressources ainsi que l'absence de gaspillage peuvent être assurées par le choix d'un sentier de consommation intertemporel optimal ${ }^{1}$, la question de l'équité intergénérationnelle n'est pas réglée pour autant (Page, 1977).

Un autre point à envisager concerne le contenu de ce qui doit être transmis aux générations futures. Autrement dit, il s'agit de se demander ce que les générations présentes doivent léguer à leurs descendants. Il est possible ici de s'appuyer sur deux approches distinctes de la durabilité du développement. La première, qualifiée de "faible ", met l'accent sur le rôle joué par le progrès technique et par les possibilités de substitution entre les différentes catégories de ressources - naturelles, techniques et humaines - dans le maintien à long terme du niveau du stock de capital global. Le maintien du stock de capital global dans le temps constitue la condition de durabilité faible.

9 Au contraire, l'autre approche qualifiée de " forte ", considère que les substitutions entre les catégories de capital et l'implication du progrès technique sont limitées de sorte que la règle de durabilité se traduit par le maintien dans le temps d'un stock de capital naturel constant (condition de durabilité forte). Le capital naturel est composé d'un ensemble d'éléments dont les fonctions sont largement complémentaires : aux côtés des ressources naturelles, figurent des services environnementaux assurés par la biosphère et les écosystèmes à travers différentes fonctions telles que le filtrage des rayons ultraviolets par la couche d'ozone, les capacités d'autoépuration et de recyclage des écosystèmes, la pollinisation des cultures... La préservation de la qualité de ces services est essentielle en ce sens qu'elle constitue une condition nécessaire à la vie sur terre. Les éléments du capital naturel pour lesquels il n'existe pas de substituts et dont la dégradation peut être irréversible constituent le capital naturel critique pour lequel des règles de sauvegarde particulières sont à prescrire.

10 Le souci environnemental tel qu'il fut pris en compte dans la définition du développement durable au sein du rapport Brundtland (1987) éclaire sans ambiguïté sur les fondements de l'éthique environnementale retenus: la nature s'enracine dans une approche anthropocentrique et elle est considérée de manière instrumentale. Ce point est fondamental pour appréhender les implications en matière de protection des ressources environnementales à long terme (cf. infra). 


\subsection{Enjeux pour la protection des ressources environnementales}

11 Les modalités de protection des ressources environnementales à long terme diffèrent selon l'approche de la durabilité retenue. Du point de vue des générations futures, l'approche de la durabilité faible considère un contenu minimaliste du capital naturel en ne prenant en compte que les ressources naturelles marchandes, et n'implique pas à leur égard de restriction particulière dans leur usage de la part des générations présentes. En effet, la durabilité du développement peut être assurée par la constitution d'un fonds de compensation provenant des rentes issues de l'exploitation des ressources épuisables. Grâce au progrès technique, les générations futures pourront utiliser ces dotations pour compenser la diminution des stocks de ressources, diminution résultant des prélèvements réalisés par les générations présentes (Hartwick, 1977 ; Solow, 1986).

D'une certaine manière, cette règle de durabilité intertemporelle traite à la fois de la distribution (globale) efficace des ressources entre générations successives et de l'éthique environnementale via la procédure de l'actualisation dans les choix économiques. L'évaluation des décisions économiques conduit à pondérer par un facteur d'actualisation les différents moments du temps. Le choix du taux d'actualisation social n'est pas neutre dans la mesure où plus sa valeur est élevée, plus le poids du présent est fort par rapport à celui accordé au futur. Ce point implique une forte asymétrie dans le traitement des générations successives: les générations futures situées sur un horizon de très long terme, supérieur à quelques décennies, seront peu prises en compte par rapport aux générations présentes. La "dictature du présent» traduit en réalité une influence tutélaire des générations présentes à l'égard des générations futures : les décisions prises par les premières expriment des choix où l'argument d'impatience est fort. Autrement dit, l'existence d'une préférence pure pour le présent implique de facto un traitement non équitable des générations successives et privilégie une utilisation des ressources naturelles pour la satisfaction des besoins présents.

Dans ce contexte, un traitement équitable entre les générations implique nécessairement un aménagement de la procédure de l'actualisation dans les choix économiques (Padilla, 2002 ; Almansa, Calatrava, 2007). Une différenciation du taux d'actualisation social sur l'horizon temporel peut être envisagée avec la diminution de la valeur du taux au fur et à mesure que l'on s'éloigne du présent afin d'accorder un poids plus grand aux générations futures. Cette différenciation peut reposer sur une décroissance du taux de préférence pure pour le présent ou sur l'absence de toute préférence pour le présent concernant les générations futures. L'absence d'unicité du taux d'actualisation social exprime à la fois le souci d'équité intra et inter générationnelle, et par là même, conditionne la manière dont l'équité environnementale peut être intégrée dans le calcul économique du décideur public. Ainsi par exemple, la gestion des déchets ménagers à long terme peut conduire à des choix technologiques différents quant aux impacts environnementaux escomptés selon la manière dont l'actualisation est réalisée. En présence de projets comportant des impacts environnementaux potentiels (pollutions diffuses dans le temps et dans l'espace, accumulation de déchets), les valeurs différentes du taux d'actualisation selon qu'il est associé au niveau intra ou intergénérationnel, la durée de l'horizon considéré (plusieurs siècles), le rôle de la croissance économique à long terme constituent des variables clés si l'on prend en compte l'équité intergénérationnelle (Ferrari, Méry, 2008). 
14 Dans une perspective utilitariste, l'existence de droits entre les générations successives légitime l'existence de transferts intergénérationnels pour assurer l'équité entre les différentes générations. C'est le cas par exemple avec l'introduction de l'éthique dans les décisions économiques avec le principe de copropriété développé par Henry (1990). Ce principe établit que deux générations successives ont un droit égal à l'existence du milieu naturel : la première arrivée sur terre ne peut exploiter irréversiblement les ressources naturelles qu'à condition d'assurer aux générations futures une compensation spécifique suffisante. L'obligation qui en découle constitue le principe qui lie les générations successives et repose sur des mécanismes institutionnels redistributifs. Les conditions d'arbitrage intergénérationnel qui déterminent les niveaux des transferts diffèrent selon que l'on considère d'une part le comportement altruiste des agents en la présence/ absence d'un planificateur et, d'autre part, l'égalité/non égalité des niveaux de consommation entre les enfants d'une même génération. En particulier, Howarth et Norgaard (1995) montrent que si la contrainte de constance des consommations intragénérationnelles est posée, alors l'intervention d'une agence publique est nécessaire, même si les agents sont altruistes : les parents ont une motivation moindre à transférer des avoirs à leurs enfants. Les parents sous-estiment le poids du bien-être de ces derniers parce qu'ils espèrent que la famille du conjoint de leur enfant sera plus riche qu'eux et pourra ainsi transférer plus d'avoirs ${ }^{2}$.

15 Au sein de l'approche de la durabilité forte marquée par la complémentarité entre les différentes ressources environnementales, des règles strictes dans l'usage des ressources naturelles sont posées afin de les préserver à long terme : taux de prélèvement inférieur au taux de renouvellement, remplacement des ressources épuisables par des ressources renouvelables, préservation totale des éléments du capital naturel critique pour lesquels aucun substitut n'existe. Ici, l'équité environnementale s'exprime par l'adoption d'un critère de durabilité nécessaire pour garantir un niveau de vie acceptable pour les générations futures.

Cette voie conduit à introduire dans l'analyse des obligations. Dans ce contexte, Page (1991) définit la durabilité non pas sur la base des préférences exprimées par les générations présentes, préférences qui détermineraient des obligations intergénérationnelles, mais sur la base d'un intérêt généralisé dans l'équité intergénérationnelle assurant la conservation des ressources sur un horizon de long terme. À la fois la conservation et la permanence des stocks de ressources naturelles doivent être garanties dans le temps. L'équité intergénérationnelle constitue alors un critère de durabilité. Dans la mesure où une allocation efficace des ressources dans le temps n'assure pas une répartition intergénérationnelle équitable des ressources ${ }^{3}$, le critère de durabilité encadre le critère d'efficience afin de garantir des niveaux de vie acceptables pour les générations futures. Cette approche considère l'éthique environnementale comme une forme d'expression particulière de l'équité intergénérationnelle déclinée en termes environnementaux : le maintien d'un niveau de bien-être dans le temps implique la préservation de la qualité des ressources ainsi que de leurs dotations in situ. La durabilité, établie hors d'un cadre utilitariste, contrairement au cas où des obligations lient les générations successives à travers l'optimisation d'une fonction de bien-être social intergénérationnelle, constitue une condition préalable à toute distribution équitable des ressources entre les générations sur un horizon de long terme. 
Cependant, au-delà de ces différentes voies susceptibles de rapprocher une distribution juste des ressources avec une éthique environnementale, il semble demeurer une difficulté majeure : celle relative au choix de la durée de l'horizon temporel. Ce point est d'importance si l'on considère qu'il existe à très long terme des incertitudes sur les dégradations environnementales, et que les choix décidés aujourd'hui intègrent difficilement ce facteur: comment prendre en compte les dommages potentiels des activités humaines dès lors que la durée de l'horizon temporel est de plusieurs siècles ? Récemment, une analyse du lien entre la valeur du taux d'actualisation et l'horizon temporel a mis en évidence deux effets contradictoires (Gollier, 2005). Un «effet richesse » associé à la croissance économique qui conduit à négliger les générations lointaines sur l'horizon temporel et un « effet précaution » qui introduit l'incertitude sur l'évolution de cette croissance. Si le premier effet invite à choisir une valeur élevée du taux d'actualisation sur l'horizon temporel et à accorder un poids significatif aux générations présentes, le second au contraire conduit à adopter un taux d'actualisation décroissant sur un très long terme : de $2,5 \%$ à $3 \%$ par an à l'horizon d'un siècle, et de $1 \%$ à $2,5 \%$ pour des horizons plus éloignés (au-delà de 500 ans).

Dans ces conditions, la protection des ressources environnementales et leur répartition dans l'espace et dans le temps suppose de se référer à une dimension du temps autre que celle de l'économie. La nature irréversible de certains phénomènes économiques à très long terme invite au respect d'une éthique environnementale dans l'élaboration des choix économiques qui est nécessairement portée par un temps long, temps qui dépasse le cadre de la décision économique strico sensu. C'est dans cette perspective que l'articulation de la durabilité du développement avec des questions mobilisant l'équité environnementale est étudiée dans le point qui suit.

\section{Distribution de la nature, protection de la nature et temps long : intérêt d'une éthique universelle de la responsabilité}

\subsection{L'éthique environnementale de Jonas : une éthique du futur}

Avec la parution en 1979 de Das Prinzip Verantwortung, les bases d'une nouvelle éthique sont jetées. A la différence de la responsabilité juridique, la responsabilité trouve maintenant sa source dans le futur («pour ce qui est à faire», Jonas, 1993, p.132) et non plus dans des obligations passées ou présentes. L'origine de ce changement d'éthique réside dans les menaces issues de la puissance de la technologie engendrée par l'homme. La limitation de l'agir humain résulte de l'obligation que nous avons à l'égard de l'avenir qui nous oblige à être responsable aujourd'hui.

L'éthique environnementale de Jonas est tout d'abord une éthique ouverte sur la biosphère et de nature englobante (Jonas, 1993). De ce point de vue, elle est un " anthropocentrisme biocentrique " et vise à la préservation de la vie sous toutes ses formes, humaines et non humaines, dans le but ultime de préserver l'humanité. Sans la nature, celle-ci ne peut perdurer. Les générations successives sont donc solidaires entre elles du fait de leur relation avec les éléments naturels : préserver la nature, c'est se donner les moyens de préserver les conditions d'existence de l'humanité, à condition que 
les actions réalisées par l'homme soient responsables, c'est-à-dire qu'elles préservent les conditions d'existence de l'humanité (Larrère, Larrère, 1997).

Dans un tel contexte, la nature de la responsabilité est directement liée au pouvoir de l'agir humain devenu dangereux pour l'espèce humaine du fait de la puissance de la technique qu'il a créée. L'homme contrôle la nature à l'aide de techniques qu'il ne contrôle pas. L'exemple le plus marquant est celui révélé par l'impact d'origine anthropique sur le climat, exemple qui révèle l'incapacité des sociétés à assurer leur responsabilité en matière environnementale (Bourg, 2003). Considérant l'existence de nombreuses incertitudes et les limites des savoirs scientifiques sur les effets futurs de nos actes (dégradations environnementales), Jonas propose de recourir à un jugement éthique soutenu par une heuristique de la peur : «La peur qui fait essentiellement partie de la responsabilité n'est pas celle qui déconseille d'agir, mais celle qui invite à agir » (Jonas, 1993, p. $300)$.

22 La peur a chez le philosophe une origine bienveillante : elle invite à passer à l'action. Les générations présentes ont le devoir d'anticiper les menaces qui découlent de leur toute puissance : l'obligation provient de l'avenir. Dès lors, la responsabilité à l'égard des générations futures est infinie dans le temps: les générations actuelles ont le devoir d'exercer une responsabilité à l'égard de leur descendance. L'intervention de l'éthique est alors légitime: c'est elle qui régule le pouvoir d'agir des individus en tant qu'être responsables de leurs actes. "Agis de façon que les effets de ton action soient compatibles avec la permanence d'une vie authentiquement humaine sur terre " (Jonas, 1993, p. 30).

Une première obligation découle du principe responsabilité. Elle s'exprime à l'égard des individus et implique à la fois les générations présentes et les générations futures dans une relation spécifique. Plus précisément, il existe une rupture temporelle des droits et obligations intergénérationnels qui fonde une équité intergénérationnelle asymétrique. Les générations présentes ont des droits et des obligations envers les générations futures parce qu'elles ont conscience de l'effet de leurs actions dans le futur, mais les générations futures ne peuvent ni revendiquer des droits, ni respecter des obligations à l'égard des générations présentes. Il y a donc une rupture avec la réciprocité qui lie traditionnellement obligations et droits.

La conséquence majeure de cette obligation réside dans l'impossibilité d'envisager la justice intergénérationnelle sur la base d'une justice redistributive entre les générations : toute ressource environnementale irréversiblement dégradée ou détruite du fait des actions humaines présentes ne pourra pas faire l'objet d'un échange intergénérationnel. Seul le recours à l'impératif jonassien permet d'éviter que les actions entravent les possibilités futures de la vie humaine sur terre : l'éthique limite ex ante les dégradations majeures de nature irréversible (Ballet, Mahieu, 2003).

Une seconde obligation, indirecte, s'exprime à l'égard de la nature et fonde la justice environnementale chez Jonas. En effet, les éléments de la nature font l'objet d'une obligation de la part des sociétés humaines parce qu'ils contribuent à la préservation des conditions d'existence de l'humanité et parce qu'ils sont dotés d'une valeur intrinsèque, indépendante de tout usage. Implicitement, les ressources environnementales sont dotées de valeurs de non-usage telles que les valeurs d'option, de legs ou d'existence. Il est important de noter que l'ensemble des valeurs associées aux éléments de la nature doit être préservé pour l'ensemble des générations. Le bien-être des générations non encore nées dépend explicitement de la qualité de l'environnement naturel : «(...) la solidarité de destin entre l'homme et la nature, solidarité nouvellement découverte à travers le danger, nous fait 
également redécouvrir la dignité autonome de la nature et nous commande de respecter son intégrité par delà l'aspect utilitaire " (Jonas, 1993, p.188).

Finalement, il existe une harmonie entre les être vivants, humains et non humains, harmonie qui ne doit pas être menacée car elle est garante de la survie des espèces en général et de celle de l'espèce humaine en particulier. La nature en tant qu'objet de la responsabilité humaine entre directement dans le champ de l'éthique. La dimension éthique de l'environnement naturel a pour fondement l'existence d'une solidarité intergénérationnelle qui est portée par le principe responsabilité chez le philosophe. La portée éthique de la responsabilité est globale dans la mesure où elle contient les interdépendances qui existent entre l'espèce humaine et les systèmes naturels. Dès lors, bien que la nature ne soit pas un sujet de droit, et qu'à ce titre elle n'a ni obligation ni devoir (droit) à l'égard de l'humanité, elle ne peut être exclue de la portée des enseignements du philosophe. En s'inscrivant sur un horizon de très long terme, à l'échelle du temps de la biosphère, Jonas propose une éthique environnementale qui interpelle les activités économiques: des changements profonds dans la sphère économique, au niveau des modes de production et des modes de consommation, sont nécessaires pour autoriser la préservation des ressources environnementales dans leur globalité (dimensions temporelle et spatiale).

\subsection{Retour aux ressources environnementales : les implications du principe responsabilité}

Dans le cadre du développement durable, il est possible de formuler une approche englobante de l'éthique environnementale à partir du principe responsabilité. L'obligation de préserver l'humanité de toute disparition constitue un impératif catégorique qui structure la pensée de Jonas. L'argument éthique, qui doit faire en sorte que l'humanité « doit être » dans un environnement viable (idée d'humanité), se traduit par une responsabilité exprimée au travers de l'équité intergénérationnelle. Une nouvelle responsabilité environnementale portée par l'équité intergénérationnelle peut alors fonder un rapport nouveau au temps dans les actes économiques dotés du pouvoir de dégradation irréversible de la nature. La crise écologique contemporaine n'est plus celle d'une nature limitée mais plutôt celle d'une nature vulnérable (Bourg, 2003). La question ici est de savoir comment articuler les différents temps en présence (temps économique et temps de la biosphère) au regard d'une éthique universelle de la responsabilité qui habite le très long terme, et d'en mesurer la portée et les limites en termes compensatoires (justice distributive).

29 Tout d'abord, il existe chez Jonas une asymétrie qui conduit à favoriser les générations futures : la question du «sacrifice» des générations présentes au profit de celles qui les suivront est posée (Bazin, 2007 ; Thévoz, 1993). Une justification possible peut trouver son origine dans la temporalité. La solidarité intergénérationnelle qui est portée par le principe responsabilité conduit Jonas à accepter l'inégalité intrinsèque qui lie les générations successives : leur appartenance à des moments distincts et irréversibles du temps leur confère une position inégale en termes de droits et de devoirs.

30 La maxime jonassienne fait référence à l'obligation de maintenir une «vie authentiquement humaine sur terre ». Cette obligation semble difficile à respecter si les modes de vie des pays développés se poursuivent et s'ils s'exportent dans les pays moins 
développés. La finitude de la planète et la menace écologique matérialisée par la vulnérabilité de la nature semblent de ce point de vue constituer deux contraintes infranchissables, même en faisant appel à l'audace du progrès technique ${ }^{4}$. Les politiques publiques doivent inciter à des changements dans les habitudes de consommation avec une contraction de celle-ci pour les pays du monde occidental. Jonas est explicite sur ce point: "Cela reviendrait à consentir à de sévères mesures de restriction par rapport à nos habitudes de consommation débridées - afin d'abaisser le niveau de vie 'occidental' de la période récente (...) dont la voracité, avec les déjections qu'elle entraîne, apparaît particulièrement coupable des menaces globales qui pèsent sur l'environnement. (...) en raison de la vérité toute simple selon laquelle une terre dont la surface est limitée n'est pas compatible avec une croissance illimitée, et qui veut que la terre ait le dernier mot » (Jonas, 1998, p. 106-107).

Le refus de la croissance est ici envisagé dans une logique d'autolimitation considérée comme un préalable à toute répartition des ressources entre les générations successives. S'il y a " sacrifice " au sens de réduction de la consommation aujourd'hui, cela concerne les générations présentes des pays développés. Dans le même temps, cela implique de revoir l'interprétation de la durabilité du développement : il s'agit en fait de considérer qu'il peut y avoir simultanément non-décroissance du bien-être et décroissance de la consommation pour les générations présentes les plus favorisées. En réalité, si la consommation de biens de base ne diminue pas dans le temps, la consommation des autres biens décroît: l'autolimitation - expression physique du «sacrifice» des générations présentes résidant dans les pays développés et obligées par un principe moral - permet aux générations présentes des pays en développement et à l'ensemble des générations futures d'accéder à un niveau de bien-être au moins égal au niveau qui assure la couverture des besoins essentiels 5 . Cette orientation permet d'entrevoir à la fois la solidarité entre les générations existantes et entre les générations successives à travers leur dépendance à l'égard de la nature: l'équité intragénérationnelle ne peut être dissociée de l'équité intergénérationnelle qui la détermine (via l'obligation de l'avenir).

Le changement majeur est ici : c'est l'obligation de l'avenir (éthique du futur) qui peut déterminer l'existence d'une compensation entre les générations présentes des plus favorisées vers les moins favorisées, et qui, parallèlement, peut définir l'effort en termes de réduction de consommation pour les générations présentes les plus favorisées (autolimitation). Le champ des actions présentes se trouve ainsi défini et délimité, et dépasse toute opposition entre l'équité intragénérationnelle et intergénérationnelle.

Dès lors, la mise en œuvre d'une éthique de la préservation des éléments de la nature qui ont la caractéristique de biens publics est nécessairement portée par la voie publique et non privée. Le contenu éthique des choix repose sur la légitimité des obligations, ce qui implique l'acceptation de normes et de règles par l'ensemble de la collectivité. La rationalité du choix du décideur devient une rationalité «collective » en ce sens que le respect par la collectivité du principe responsabilité dépend avant tout de sa reconnaissance sociale. "Le savoir, le vouloir et la puissance sont collectifs, leur contrôle doit donc l'être également: seuls les pouvoirs publics peuvent l'exercer, et cela nécessite finalement un large accord à la base » (Jonas, 1998, p.105).

34 Compte tenu des menaces exercées par l'usage des ressources énergétiques fossiles à l'échelle de la biosphère, la mise en œuvre du principe responsabilité doit conduire à l'évitement d'une catastrophe potentielle dans un univers d'incertitudes. On peut considérer une application selon deux niveaux qui se superposent: une redistribution (transferts) entre générations présentes sur un temps court, et le legs d'une nature 
préservée sur un temps long, legs rendu possible parce qu'une autolimitation des actions présentes via le principe responsabilité a été introduite dans la sphère des activités humaines. C'est à ce prix qu'il est possible de respecter l'intégrité des éléments de la nature et d'espérer préserver la survie de l'humanité.

\section{Conclusion}

Un tel positionnement moral de Jonas, assis sur le principe responsabilité, ne peut être imposé comme une contrainte à autrui : il demeure volontaire. C'est là certainement une difficulté de taille (Bazin, 2007). Jonas a conscience de cela et reconnaît qu'il n'est pas facile de mettre en œuvre des politiques accompagnant les mesures de restriction nécessaires et de les faire accepter de tous. L'heuristique de la peur peut orienter la conscience collective et faire en sorte que la nature, essentielle à la vie humaine, soit préservée.

Cette voie suppose au préalable une révision des préférences des personnes détachées d'une emprise matérialiste forte afin d'envisager le renoncement à certains biens pour en choisir d'autres, en toute liberté! «Faire avec moins » constitue une restriction morale qui traduit l'existence d'une responsabilité environnementale chez l'individu : accepter la réduction non pas du bien-être mais du confort attaché à l'usage de certains biens dont la production et/ou la consommation engendre des dégradations de nature irréversible de l'environnement naturel. Cette posture relativise fortement le rôle du progrès technique et la place que ce dernier occupe dans le développement des sociétés. Reléguer la technologie au second plan des valeurs dans les civilisations "modernes" bouscule nécessairement l'attachement inconscient des choix de vie portés par la croissance économique. Et pourtant, c'est de cette emprise qu'il faut urgemment se libérer pour concevoir autrement la satisfaction des besoins des générations présentes et futures dans un environnement préservé.

\section{BIBLIOGRAPHIE}

Almansa C., Calatrava J., 2007, « Reconciling sustainability and discounting in cost-benefit analysis: a methodological proposal », Ecological Economics, 60, p. 712-725.

Azqueta D., Delacamara G., 2006, « Ethics, economics and environmental management », Ecological Economics, 56, p.524-533.

Ballet J., Mahieu F.-R., 2003, Ethique économique, Collection mise au point, Ellipses, Paris.

Barrett S., 2008, "The incredible economics of Geoengineering », Environmental and Resource Economics, 39 (1), p. 44-54.

Bazin D., 2007, Sauvegarder la nature. Une introduction au Principe Responsabilité de Hans Jonas, Éditions Ellipses, Paris.

Bourg D., 2003, Le nouvel âge de l'écologie, Ed. Charles Léopold Mayer. 
Brundtland G. H., 1987, Our common future, World Commission on Environment and Development, Oxford.

Dasgupta P. 2008, « Nature in economics », Environmental and Resource Economics, 39 (1), p. 1-7.

Dasgupta P.S., Heal G.M., 1979, Economic theory and exhaustible resources, Cambridge university press, Cambridge.

Ferrari S., Méry J., 2008, « Equité intergénérationnelle et préoccupations environnementales. Réflexions autour de l'actualisation. », Management et Avenir, 20, p. 240-257.

Gagnon B., Lewis N., Ferrari S., 2008, « Environnement et pauvreté : regards croisés entre l'éthique et la justice environnementales ", Écologie et Politique, 35, p.79-90.

Gollier C., 2005, « Quel taux d'actualisation pour quel avenir ? », Revue Française d'Economie, 19 (4), p. 59-81.

Gosseries A., 2008, «Les théories de la justice intergénérationnelle. Synopsis à l'usage des durabilistes pressés ", Raison Publique, $n^{\circ} 8$, accessible via le lien : http://www.raison-publique.fr/ Les-theories-de-la-justice.html

Hartwick J. M., 1977, « Intergenerational equity and the Investing of Rents from Exhaustible Resources », The American Economic Review, 67, p. 972-974.

Henry C., 1990, «Efficacité économique et impératifs éthiques : l'environnement en copropriété », Revue Economique, 2, p. 195-214.

Howarth R. B., Norgaard R. B., 1995, « Intergenerational choices and global environmental change » in Bromley D.W., The Handbook of environmental economics, Blackwell Publishers, Oxford. Jonas H., 1993, Le principe responsabilité, une éthique pour la civilisation technologique, traduction de Das Prinzip Verantwortung (1979), troisième édition, Éditions du Cerf, Paris.

Jonas H., 1998, Pour une éthique du futur, Éditions Payot et Rivages, Paris.

Larrère C., 1997, Les philosophies de l'environnement, Presses Universitaires de France, Paris, 124 pages.

Jouvet P.-A., Michel P., Vidal J.-P., 2000, «Intergenerational Altruism and the Environment », Scandinavian Journal of Economics, 102 (1), p. 135-150.

Larrère C., Larrère R., 1997, Du bon usage de la nature, Édition Aubier, Paris.

Mikkelson G. M, Gonzalez A, Peterson G. D., 2007, « Economic Inequality Predicts Biodiversity Loss », PLoS ONE 2 (5), e444, http://www.plosone.org/article/info\%3Adoi\%2F10.1371\% 2Fjournal.pone.0000444

Padilla E., 2002, « Intergenerational equity and sustainability », Ecological Economics, 41 (1), p. 69-83.

Page R.T., 1977, Conservation and economic efficiency. An approach to materials policy, Johns Hopkins University Press, Baltimore, MY, USA.

Page R. T., 1991, «Sustainability and the problem of valuation », in Costanza R. (ed.), Ecological economics: the science and management of sustainability, Columbia University press, New York, $\mathrm{p}$. 58-74.

Rawls J., 1971, A Theory of Justice, Harvard University Press, Cambridge, Mass., trad. française 1987, Seuil, Paris, 
Solow R., 1986, « On the intergenerational allocation of natural resources », Scandinavian Journal of Economics, 88 (1), p. 141-149.

Thévoz J.-M., 1993, « Générations présentes, generations futures, quelles priorités ? », in Baertschi B., Foppa C., Mauron A., Müller D., Simon R., Thévoz J.-M., Nature et descendance, Hans Jonas et le principe "Responsabilité », Labor et Fides, Collection Le champ éthique, Genève, Volume 25, p.73-84.

WCED (World Commission on Environment and Development), 1987, Our common future, Brundtland report, Oxford University Press, Oxford.

\section{NOTES}

1. Cela est vérifié si les éléments considérés sont correctement évalués par les marchés, ce qui n'est pas toujours le cas en présence d'externalités et/ou de défaillances du marché. La question de l'évaluation des services environnementaux offerts par les écosystèmes et la biosphère est délicate notamment du point de vue des méthodes utilisées pour révéler les préférences des agents économiques. Sur ce point, le lecteur peut se reporter à (Azqueta, Delacamara, 2006).

2. On peut noter que si l'altruisme lie les générations successives, alors les générations présentes peuvent également participer à l'amélioration du bien-être des générations futures en réalisant des dépenses destinées à améliorer la qualité de l'environnement (Jouvet et al., 1997).

3. Sur cette question, se reporter à Dasgupta et Heal (1979).

4. Le progrès technique est souvent avancé comme solution à de nombreux maux dont souffre l'humanité. Récemment, des techniques de contrôle du climat visant à limiter le rayonnement solaire ont été avancées pour lutter contre le changement climatique. Sur ce point, se reporter à (Barrett, 2008).

5. On peut par exemple faire référence aux biens premiers chez Rawls (1971).

\section{RÉSUMÉS}

L'objet de cet article vise à étudier le développement durable sous l'angle de l'éthique environnementale. En particulier, on s'interroge sur la nécessité d'introduire dans l'analyse économique une justice d'autolimitation comme préalable à toute répartition des ressources environnementales entre les générations successives. Dans cette perspective, et en considérant les effets à long terme des actions humaines sur la nature, les conditions de mise en œuvre d'une éthique du futur sont analysées à travers le principe Responsabilité de H. Jonas.

This article aims to study the sustainable development through the environmental ethics dimension. First, we analyse why a self-restriction justice has to be included into the economic analysis before any allocation of the environmental resources between generations. As the impacts of human actions on nature are dealing with the long run, we finally discuss the conditions for implementing an ethics of the future based on the Responsibility principle of $\mathrm{H}$. Jonas. 
INDEX

Keywords : discounting, environmental ethics, intergenerational equity, Jonas, Responsibility principle, sustainable development

Mots-clés : actualisation, développement durable, équité intergénérationnelle, éthique environnementale, Jonas, principe Responsabilité

\section{AUTEUR}

\section{SYLVIE FERRARI}

Sylvie Ferrari, maître de conférences HDR en économie à l'Université Bordeaux IV, est membre du GREThA - UMR CNRS 5113. Ses enseignements sont principalement rattachés à l'économie de l'environnement et du développement durable. Ses recherches concernent les thématiques suivantes : la durabilité environnementale de la production, l'éthique environnementale et le développement durable, la gestion durable des ressources en eau, la multifonctionnalité de l'agriculture. Plus récemment, son programme de recherche a développé une investigation sur la question du rôle de l'éthique environnementale dans les décisions économiques en s'appuyant sur les travaux du philosophe Hans Jonas (Ferrari S., Mery J. (2008), « Equité intergénérationnelle et préoccupations environnementales. Réflexions autour de l'actualisation », Management et Avenir, n²0, p. 240-257 ; Gagnon B., Lewis N., Ferrari S. (2008), « Environnement et pauvreté : regards croisés entre l'éthique et la justice environnementales ", Écologie et Politique, n³5, p.79-90). GREThA - UMR CNRS 5113, Université Bordeaux IV, sylvie.ferrari@u-bordeaux4.fr 